\title{
La rimozione percutanea di fibrina (Percutaneous Fibrin Sleeve Stripping) dai cateteri venosi centrali permanenti per emodialisi può essere causa di tromboembolia polmonare asintomatica
}

\author{
G. Bergonzi ${ }^{1}$, M. Bacchi ${ }^{1}$, A. Ballocchi ${ }^{1}$, F. Fontana ${ }^{1}$, PG. Poisetti ${ }^{1}$, R. Scarpioni ${ }^{1}$, \\ L. Cristinelli' ${ }^{1}$ P. Quaretti', M. Rago ${ }^{2}$, GL. Moschini' ${ }^{2}$,L. Cerri ${ }^{3}$, M. Mori ${ }^{3}$ \\ ${ }^{1}$ Divisione Nefrologia e Dialisi, \\ ${ }^{2}$ Servizio di Radiologia, \\ ${ }^{3}$ Servizio di Medicina Nucleare Ospedale Civile Piacenza
}

egli ultimi anni la rapida crescita del programma di trattamento dialitico extracorporeo dell'insufficienza renale cronica è stata accompagnata dall'aumento proporzionale dei problemi connessi alla creazione e conservazione di un accesso vascolare (1).

Se la creazione di una fistola arterovenosa (FAV) rappresenta l'opzione preferenziale, l'immissione in emodialisi di pazienti ultraottuagenari, o con patologie cardiache rilevanti che mal tollererebbero la presenza di una FAV prossimale, e di pazienti che col protrarsi della loro vita dialitica esauriscono il patrimonio vascolare periferico, hanno contribuito ad incrementare la percentuale di pazienti che eseguono il trattamento emodialitico utilizzando un catetere venoso centrale tunnellizzato (CVCT) quale accesso vascolare.

Il sito preferenziale di inserzione di un CVCT è rappresentato dalla vena giugulare interna destra in quanto tale sede è gravata da un minor numero di complicanze immediate (legate alla tecnica di posizionamento del catetere stesso) e tardive (secondarie al traumatismo che il catetere produce sulla parete dei vasi con formazioni di stenosi) (2).

I CVCT hanno, rispetto agli altri accessi vascolari, alcuni vantaggi:

a) sono immediatamente utilizzabili non richiedendo un tempo di maturazione e sviluppo come le fistole artero-venose con vasi nativi o in PTFE;

b) non comportano conseguenze emodinamiche;

c) sono utilizzabili nella totalità dei pazienti in quanto diversi sono $\mathrm{i}$ siti di posizionamento;

d) non richiedono l'utilizzo di aghi, cosa che li rende graditi ai pazienti anziani o comunque ai pazienti che mal tollerano la venipuntura;

e) sono facilmente sostituibili.

Gli svantaggi dei CVCT rispetto agli altri accessi vascolari comprendono:

a) elevata morbidità secondaria a trombosi ed infezioni;

b) rischio di stenosi ed occlusioni di vene centrali;

c) flusso ematico spesso inferiore a quello fornito da una FAV;

d) ricircolo più elevato;

e) possibile scarsa accettazione per motivi estetici.

La complicanza che più frequentemente si osserva è la formazione di un trombo nel lume del catetere con limitazione assai importante del flusso ematico durante la dialisi. 


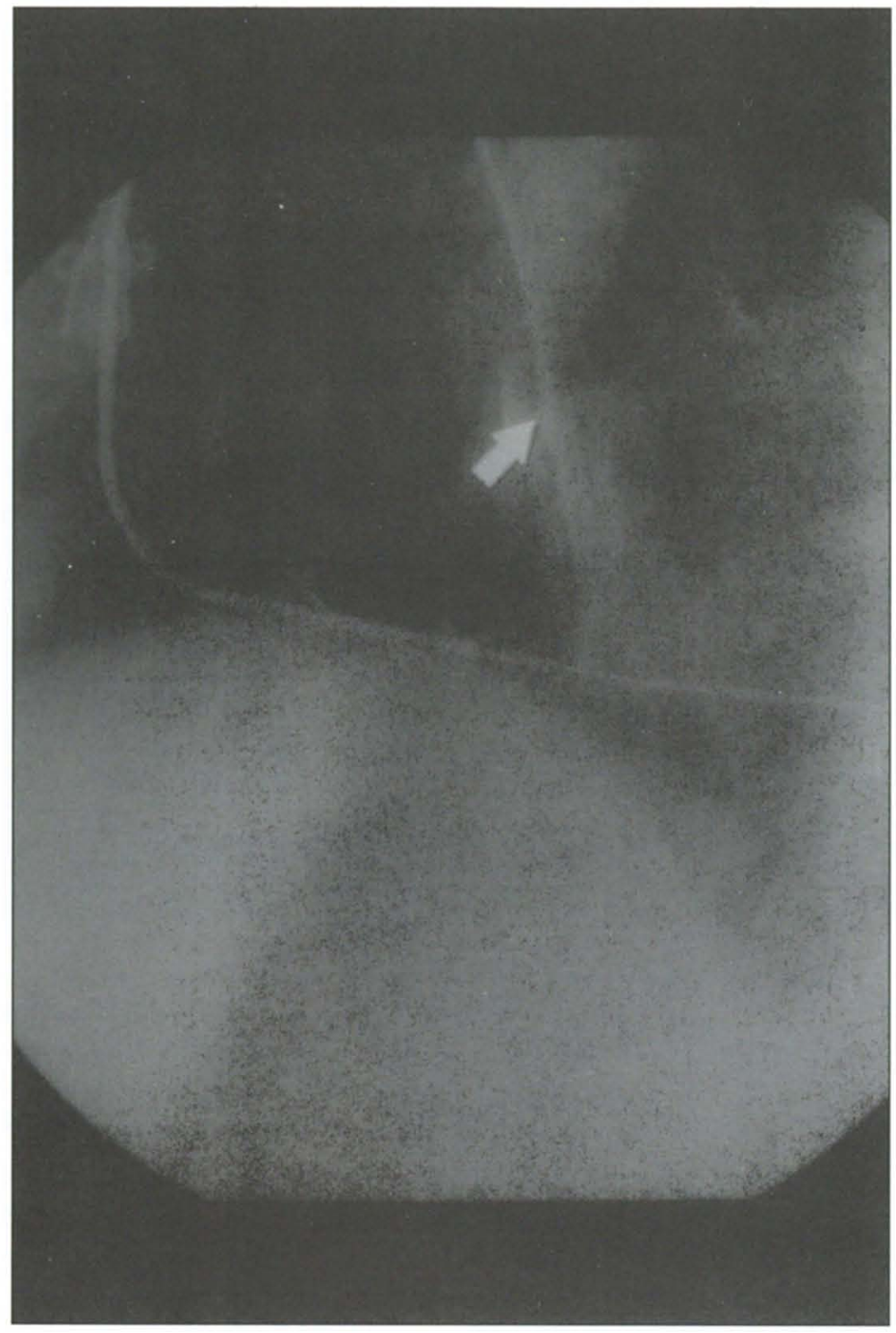

Fig. 1 - Irregolarità "a manicotto" del margine del catetere venoso centrale.

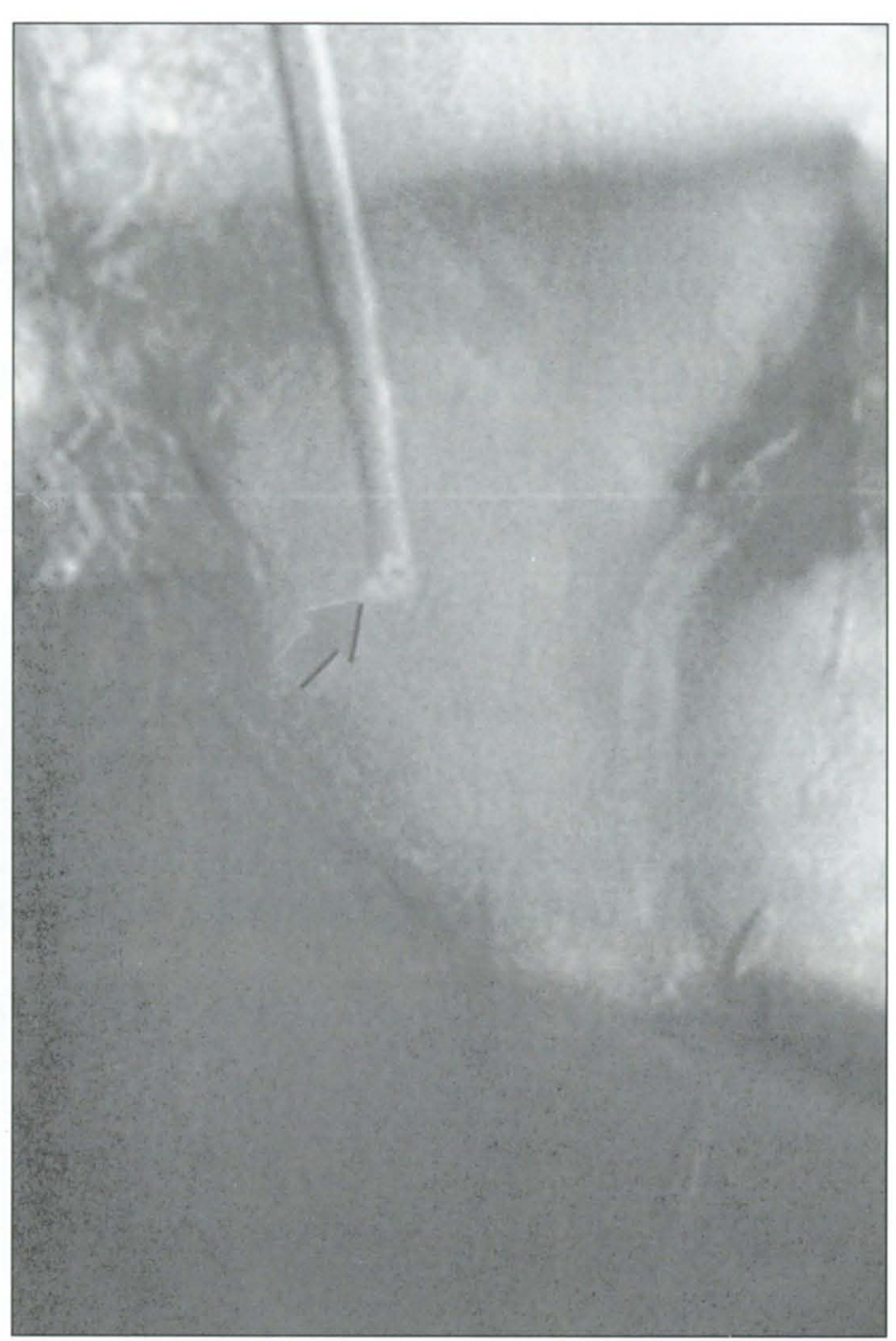

Fig. 2 - Formazione di fibrina in prossimità del foro terminale del venoso centrale (angiografia digitale).
La somministrazione locale di urokinasi in volume sufficiente a riempire il lume del catetere e lasciata in situ per un tempo variabile da 10' a 30' è il più delle volte risolutiva nel ripristinare la pervietà del catetere.

Non sempre, però, l'impiego di urokinasi anche in dosi crescenti e per tempi più prolungati, è in grado di ristabilire il funzionamento del catetere.

La deposizione di fibrina sulla superficie esterna del CVCT spiega il possibile insuccesso che si può osservare dopo infusione locale di urokinasi e la positiva risposta all'infusione sistemica del farmaco protratta oltre le 12 ore (3).

Qualora l'infusione di urokinasi non sia stata in grado di ripristinare la pervietà del CVCT è indicato lo stu- dio radiologico con mezzo di contrasto, anche se non sempre tale indagine è risolutiva.

I segni radiologici della deposizione di fibrina possono (4), comunque, essere rappresentati da:

- riduzione del flusso del mezzo di contrasto dai fori terminali;

- irregolarità dei margini del CVCT (Fig. 1, 2);

- reflusso del mezzo di contrasto con immagine a binario lungo le branche del CVCT.

Una metodica alternativa o sequenziale all'uso di farmaci fibrinolitici è rappresentata dal "Percutaneous Fibrin Sleeve Stripping" (PFSS) illustrata recentemente da Crain MR et al (4).
La metodica prevede la rimozione meccanica mediante un catetere a cappio inserito nella vena femorale dx della fibrina depositata sulla superficie esterna dei CVCT (Fig. 3). In letteratura non sono descritte complicanze tromboemboliche secondarie alla tecnica, ma non sono stati eseguiti studi specifici per escludere tale eventualità.

\section{Pazienti e metodi}

Sei pazienti in trattamento emodialitico cronico in media da $65 \pm 52$ mesi portatori di CVCT in vena giugulare interna $\mathrm{dx}$ che avevano presentato flusso ematico intradiali- 
tico inadeguato $(<200 \mathrm{ml} / \mathrm{min})$ anche dopo trattamento locale con urokinasi sono stati sottoposti a PFSS. I cateteri (Soft-Cell, Vas$\mathrm{Cath}^{\circledast}$ ) erano posizionati in vena giugulare interna $\mathrm{dx}$ da $10.6 \pm 5$ mesi al momento dello PFSS.

\section{Tecnica}

In accordo con gli Autori (3) la procedura di PFSS era preceduta da controllo fluoroscopico per evidenziare eventuali cause non trombotiche del cattivo funzionamento del catetere, quali inginocchiamenti, e per valutare la posizione del tratto terminale del catetere ed i suoi rapporti anatomici.

Veniva, quindi, eseguita indagine angiografica con mezzo di contrasto

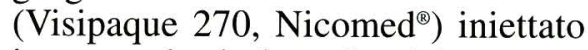
in entrambe le branche del catetere alla velocità di infusione di 10 $\mathrm{ml} / \mathrm{min}$ per un volume di $25 \mathrm{ml}$.

La tecnica originariamente descritta (3) veniva modificata nel modo seguente: veniva iniettato mezzo di contrasto anche attraverso un catetere femorale Pig Tail $5 \mathrm{~F}$ fatto risalire lungo la vena cava inferiore sino ad incontrare il catetere venoso centrale. Dopo infiltrazione dei tessuti con carbocaina veniva posizionato con tecnica di Seldinger un introduttore vascolare (Terumo ${ }^{\circledR 7} \mathrm{~F}$ ) in vena femorale $\mathrm{dx}$ attraverso il quale era introdotto un catetere a cappio $(6 \mathrm{~F}$ Snare Catheter, AmplazMicrovena ${ }^{\circledR}$ ) che veniva fatto risalire lungo la vena cava inferiore (Fig. 4).

Quindi veniva introdotta attraverso la branca venosa del catetere per emodialisi una guida idrofilica standard $\left(\right.$ Terumo $\left.^{\circledR}\right)$ spinta fino ad incontrare il catetere a cappio.

Il catetere a cappio veniva fatto risalire seguendo la guida lungo la superficie esterna del CVCT fino al tronco comune della vena brachiocefalica (Fig. 5, 6).

Il catetere a cappio veniva quindi stretto attorno al CVCT e veniva ritirato delicatamente lungo la superficie esterna fino ai fori terminali delle due branche.

Tale manovra di "stripping " era eseguita più volte.

Fig. 3 - Il catetere a cappio, la guida, il catetere venoso centrale.

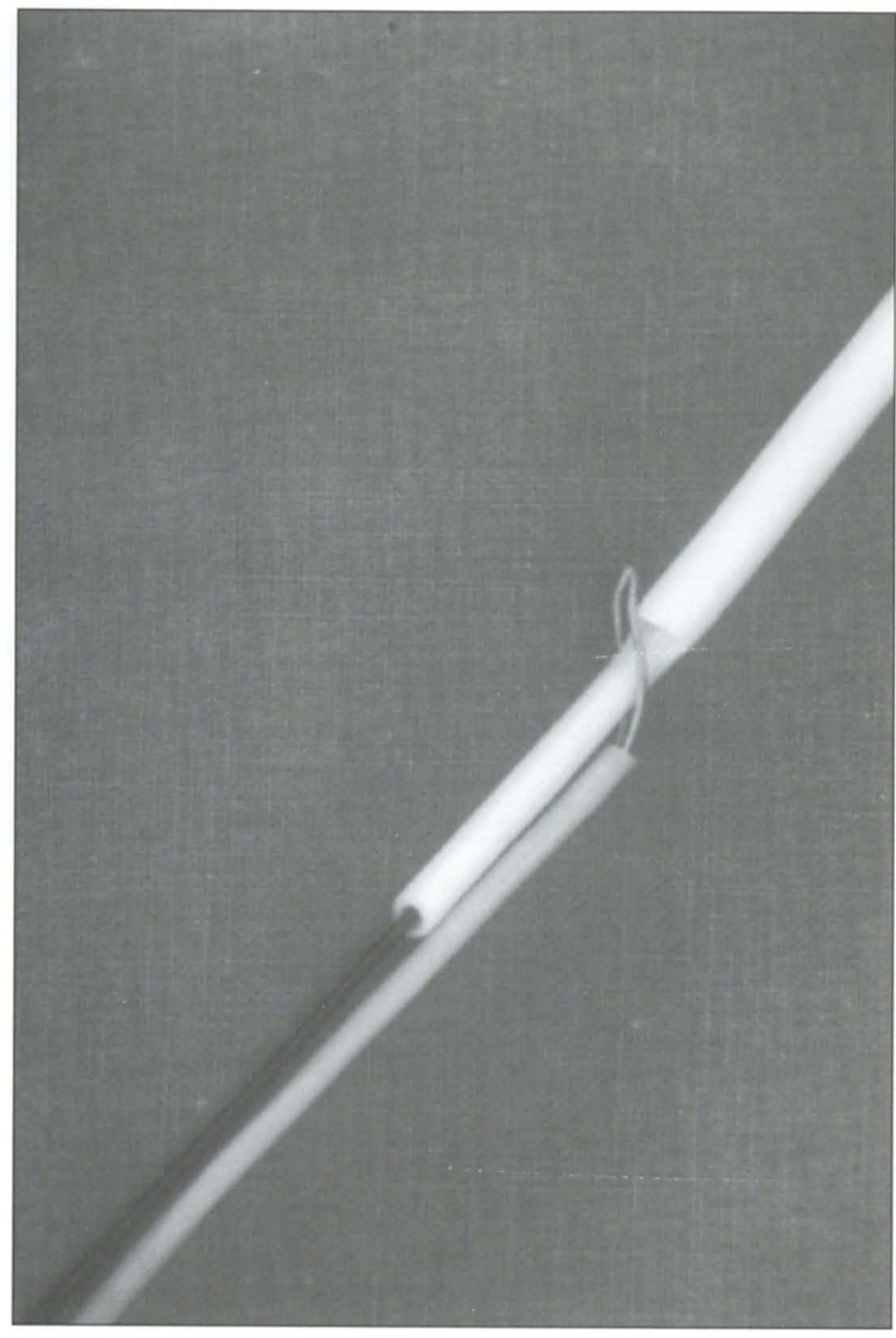

Per verificare eventuali episodi di tromboembolia polmonare asintomatica secondari al distacco di formazioni di fibrina dal CVCT ed al loro passaggio nel circolo polmonare i pazienti venivano sottoposti il giorno precedente l'esecuzione di PFSS e subito dopo l'intervento a scintigrafia polmonare con $99 \mathrm{mTc}$.

\section{Risultati}

La metodica, eseguita in regime ambulatoriale, era ottimamente tollerata ed un solo paziente presentò tosse durante PFSS.

In 5 pazienti si otteneva ripristino di un adeguato flusso ematico intradialitico del CVCT, mentre in un paziente si rendeva necessaria la sostituzione del CVCT che, peraltro, esaminato dopo la sua rimozione non rivelava trombosi endoluminale o deposizione di fibrina sulla superficie esterna in prossimità dei fori terminali.

La scintigrafia polmonare eseguita dopo PFSS dimostrava in 5 pazienti deficit di captazione subsegmentaria del tracciante secondari a microtromboembolia polmonare clinicamente asintomatica (Fig. 7, 8).

\section{Discussione}

I CVCT rappresentano l'ultima tappa della ricerca relativa agli accessi vascolari nei pazienti in trattamento dialitico cronico iniziata nel 1960 da Quinton con l'introduzione dello shunt artero-venoso esterno. In seguito al diffondersi della dialisi sca- 


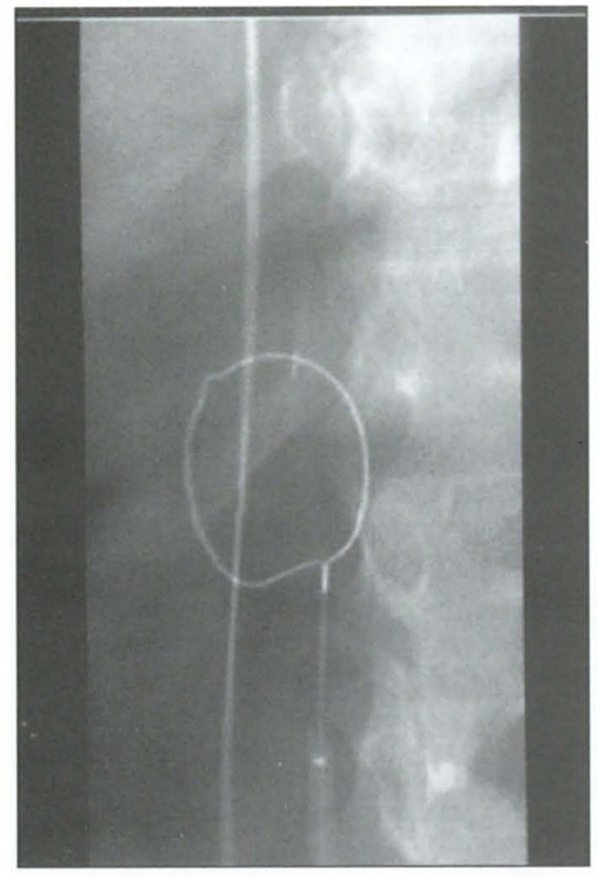

Fig. 4 - Il catetere a cappio in vena cava inferiore risale lungo la guida introdotta attraverso il catetere venoso centrale.

turì l'esigenza di un accesso vascolare di più agevole utilizzo e di maggior durata. Nel 1966 Brescia e Cimino svilupparono la tecnica chirurgica per la creazione della fistola artero-venosa. Negli anni '70 si diffuse la tecnica di allestire le fistole artero venose con interposizione di segmenti di protesi vascolari costituite da diversi materiali (5).

La fine degli anni ' 80 e il decorso degli anni ' 90 con la disponibilità di materiali vascolari biocompatibili vedono una progressiva diffusione dell'utilizzo dei CVCT come accesso vascolare per il trattamento emodialitico a medio-lungo termine: particolarmente per pazienti in attesa di maturazione della fistola artero-venosa o di consolidamento dei processi di guarigione in sede di catetere peritoneale; per pazienti sottoposti a ripetuti trattamenti plasmaferetici; per pazienti cardiopatici che necessitano di trattamenti di ultrafiltrazione per mantenere un adeguato compenso emodinamico.

I CVCT rappresentano anche la soluzione definitiva per quei pazienti che hanno esaurito il loro patrimonio vascolare periferico, pazienti solitamente destinati a tentativi di chirur-
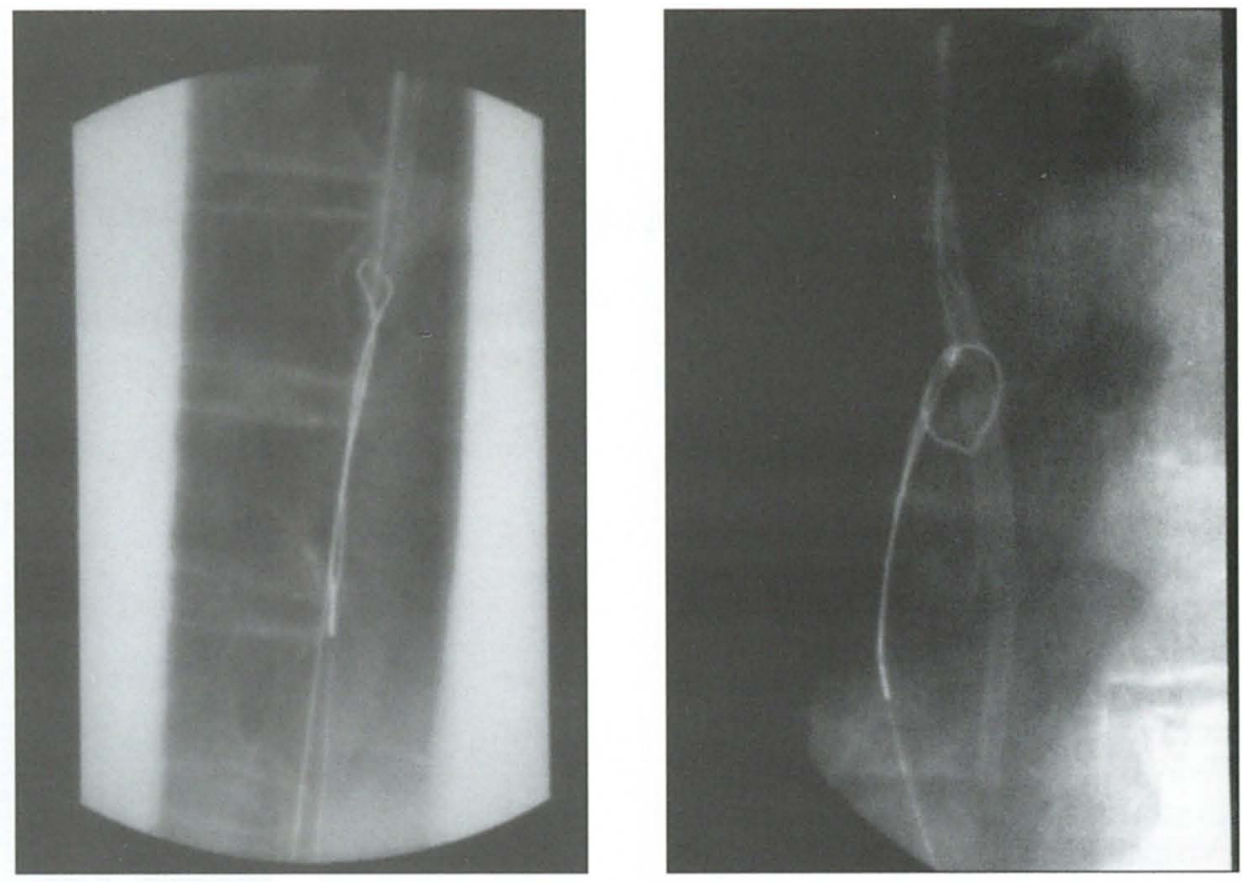

Fig. 5-6 - Il catetere a cappio risale lungo la guida e lungo la superficie esterna del catetere venoso centrale.

gia vascolare "estrema" (di cosiddetto terzo livello).

Accanto a questi trovano una indicazione al posizionamento di CVCT pazienti che hanno una aspettativa di vita limitata, pazienti uremici con grave compromissione della funzione ventricolare sinistra ed infine pazienti che non tollerano il dolore legato alla puntura della FAV.

Negli Stati Uniti durante il 1996 il $19 \%$ dei pazienti in trattamento emodialitico a 2 mesi dall'inizio della dialisi utilizzava un CVCT, mentre tale percentuale era pari al $10 \%$ nel 1993 (1).

Jean et al (6) in un recente lavoro riportano una percentuale di CVCT pari al $15 \%$ di tutti gli accessi vascolari adottati nel loro centro. I dati relativi alla sopravvivenza dei CVCT sono assai variabili.

Questo è dovuto alle diverse sedi di posizionamento dei CVCT, alle diverse indicazioni cliniche che sono alla base della decisione del loro posizionamento, ai diversi tipi di CVCT oggi disponibili: differenti per materiali, tipo di cuffia, numero delle cannule, numero dei lumi, geometria del lume. La "patency rate" varia da 30 a $74 \%$ ad un anno e da 30 a $43 \%$ a due anni
(7-11) secondo le diverse casistiche. La causa più frequente di insuccesso dei CVCT fra le complicanze tardive (cioè insorte dopo 1 mese dal posizionamento) è la comparsa di infezione batterica. Tuttavia si stima che una percentuale pari al 2-9\% di CVCT venga rimossa tardivamente per complicanze trombotiche condizionanti un insufficiente flusso ematico intradialitico $(8,9,11)$.

La formazione di fibrina all'interno del lume del CVCT può essere il nucleo di aggregazione per la formazione di trombi occludenti il lume del catetere. Questi sono normalmente rimossi con una energica aspirazione o con l'infusione locale di urokinasi.

Causa di insufficiente funzionamento dei CVCT può essere anche la deposizione di fibrina sulla superficie esterna in prossimità dei fori terminali (Fig. 2); tali formazioni possono funzionare come valvole permettendo l'infusione, ma non l'aspirazione (12).

La deposizione di fibrina sulla superficie esterna del catetere può iniziare dal sito di inserzione sede di lesione intimale $\mathrm{o}$ in prossimità della parte terminale del catetere quando sia a contatto della parete venosa ed inizierebbe già nelle prime 24 ore dopo il 


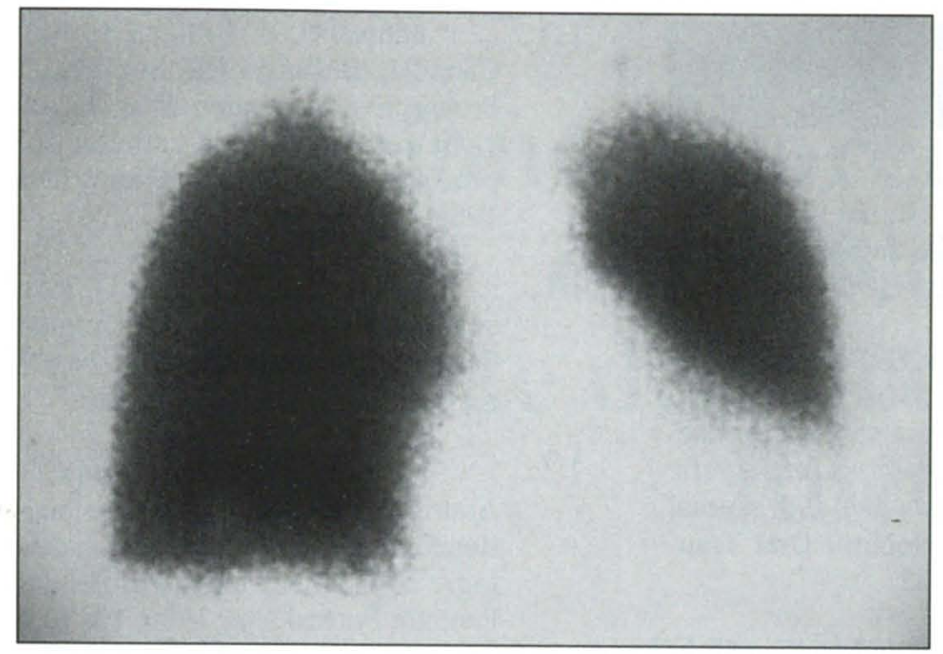

Fig. 7a - Scintigrafia polmonare precedente all'esecuzione di PFSS.

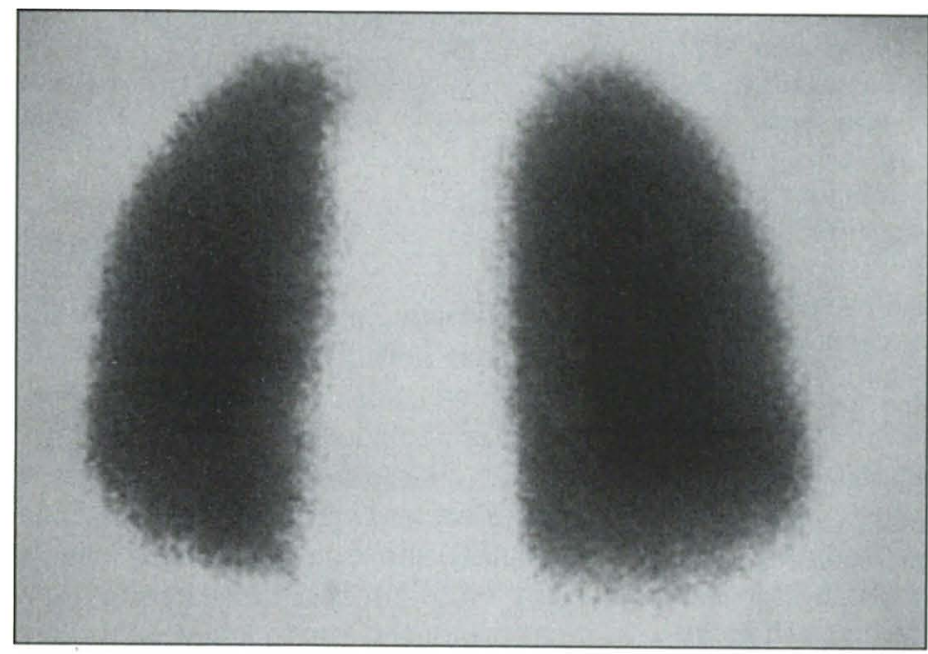

Fig. 8a - Scintigrafia polmonare precedente all'esecuzione di PFSS.

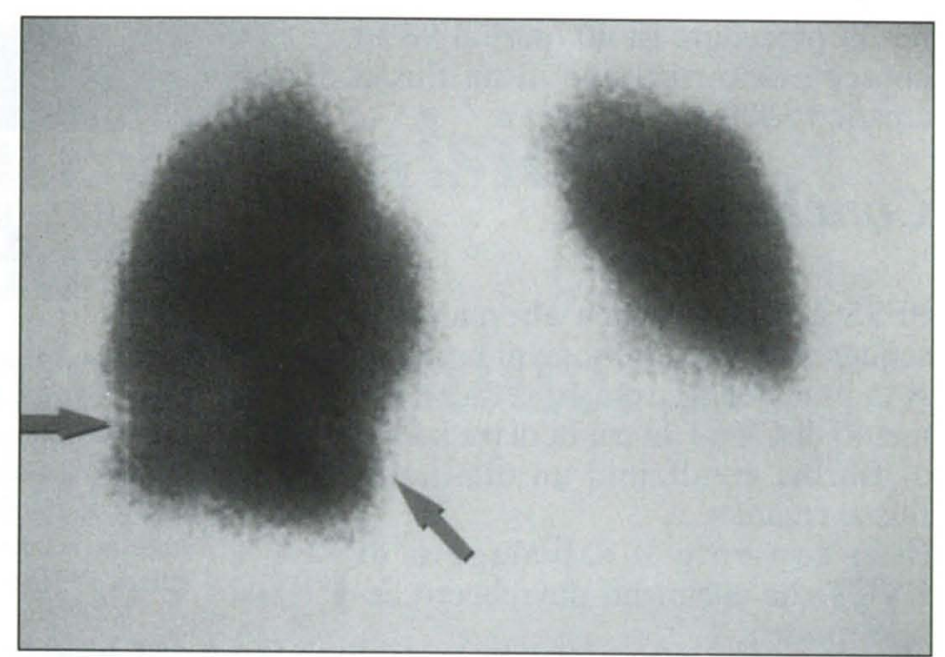

Fig. 7b - Scintigrafia polmonare successiva all'esecuzione di PFSS. Le frecce indicano i deficit di captazione del tracciante radioattivo.

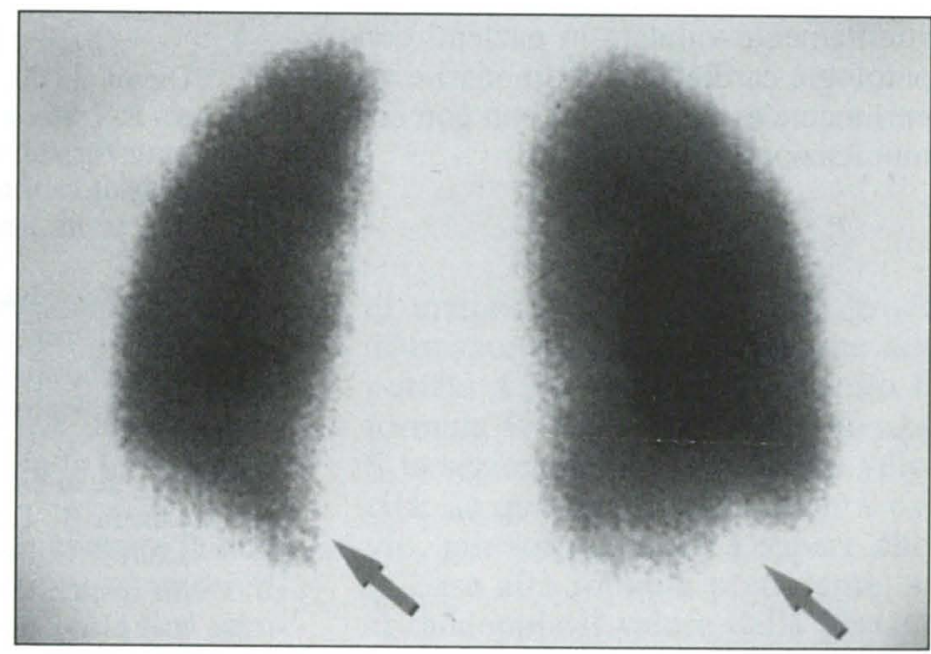

Fig. 8b - Scintigrafia polmonare successiva all'esecuzione di PFSS. Le frecce indicano i deficit di captazione del tracciante radioattivo. posizionamento per avvolgere nel giro di di 5-6 giorni tutto il catetere (13). Ciononostante è abbastanza raro osservare formazioni di fibrina sulla superficie esterna dei CVCT quando vengono rimossi, poiché la fibrina rimane adesa alla parete venosa.

L'ulteriore passo per ripristinare la funzione perduta senza dover ricorrere alla sostituzione del CVCT è rappresentato dalla rimozione meccanica della fibrina mediante PFSS.

Questa tecnica inserita dal 1997 nelle linee guida del National Kidney Foundation -DOQI è accreditata di una totale mancanza di complicanze non essendo mai stati osservati finora episodi di tromboembolia nonostante la metodica possa provocare il distacco dalla superficie del catetere di frammenti di fibrina.

Anche la rimozione di CVCT può, tuttavia, provocare la formazione di emboli di fibrina senza indurre, peraltro, episodi di tromboembolia polmonare sintomatica. A conferma di questa ipotesi vi è l'osservazione che in CVCT rimossi per flusso ematico inadeguato, assai raramente si osservano formazioni di fibrina che, evidentemente, passano in circolo al momento della rimozione del CVCT. L'indagine mediante scintigrafia con $99 \mathrm{mTc}$ della perfusione polmonare da noi eseguita prima ed immediatamente dopo PFSS ha comunque con- fermato l'ipotesi che il distacco della fibrina dai CVCT causa episodi di tromboembolia seppur clinicamente non rilevanti.

Infatti la scintigrafia polmonare con $99 \mathrm{mTc}$ ha evidenziato in 5 dei 6 pazienti deficit di captazione subsegmentaria del tracciante secondari a tromboembolia polmonare.

Nella nostra iniziale esperienza la metodica, ben tollerata dai pazienti ed eseguita in regime ambulatoriale, si è dimostrata risolutiva nel ripristinare un flusso ematico ottimale in 5 dei 6 pazienti sottoposti a PFSS.

Crain et al riportano una casistica di 40 PFSS eseguite su 23 CVCT senza alcun episodio di embolia polmona- 
re; 39 procedure su 40 (pari al 98\%) conseguivano ripristino di un flusso ematico $>300 \mathrm{ml} / \mathrm{min}$ (4).

\section{Conclusioni}

PFSS è una metodica alternativa o sequenziale alla infusione di urokinasi in grado di ripristinare il funzionamento di CVCT in cui la deposizione di fibrina condizioni un diminuito flusso ematico.

Essa consente il salvataggio di CVCT che altrimenti dovrebbero essere rimossi.

Nella nostra esperienza può provocare microemboembolia polmonare clinicamente asintomatica.

Il suo utilizzo deve essere, pertanto, attentamente valutato in pazienti con patologie cardiache e polmonari e sicuramente escluso in pazienti con comunicazioni interatriali.

\section{BIBLIOGRAFIA}

1. Hakim R, Himmelfarb J. Hemodialysis access failure: A call to action. Kidney Int 1998; 54: 1029.

2. Schillinger F, Schillinger D, Montagnac R, Milcent T. Post catheterisation vein stenosis in haemodialysis: comparative angiografic study of 50 subclavian and internal jugular access. Nephrol Dial Transplant 1991; 6: 722.

3. Vogt K, Tillmann U, Blumberg A. Successful fibrinolysis in permanent hemodialysis catheter obstruction. Nephron 1987; 45: 174.

4. Crain MR, Mewissen MW, Ostrowski GJ, Paz-Fumagalli R, Beres RA, Wertz R. Fibrin sleeve stripping for salvage of failing hemodialysis catheters: technique and initial results. Radiology 1996; 198: 41.

5. Kapoian T, Sherman RA. A bief history of vascular access for hemodialysis: An unfinished story. Semin Nephrol 1997; 17: 239.

6. Jean $\mathrm{G}$, Charzot $\mathrm{C}$, Vanel $\mathrm{T}$, Charra B, Terrat JC, Calemard E, Laurent G. Central venous catheters for hemodialysis: looking for optimal blood flow. Nephrol Dial Trasplant 1997; 12 (8): 1689.

7. Suchoki P, Comìnlon P, Knelson M. Silastic cuffed catheters for hemodialysis vascular access: thrombolytic and mechanical correction of HD catheters malfunction. Am J Kidney Dis 1996; 28: 239.

8. Moss AH, Vasilakis C, Holley JL, Foulks CJ, Pillai K, Mc Dowell DE. Use of a silicone dual-lumen catheter with a Dacron cuff as a long-term vascular access for hemodialysis patients. Am J Kidney Dis 1990; 16: 211.

9. Canaud B, Beraud JJ, Joyeux H. Mion C. Internal jugular vein cannulation with two silicone rubber catheters: A new and safe temporary vascular access for hemodialysis. Thirty months' experience. Artif Organs 1986; 10: 397.
10. Schwabb SJ, Buller GL, McCann RL, Bollinger RR, Stickel DL. Prospective evaluation of a Dacron cuffed hemodialysis catheter for prolunged use. Am J Kidney Dis 1988; 11 : 166.

11. Gibson SP, Mosquera D. Five years experience with the Quinton Permcath for vascular access. Nephrol Dial Transpant 1991; 6: 269.

12. Schneider TC, Krzymda E, Andris D, Quebbeman EJ. The malfunctioning Silastic catheter: radiologic assessment and treatment. J Parenter Enteral Nutr 1986; 10: 70.

13. Hoshal VL, Ause RG, Hoskins PA. Fibrin sleeve formation on indwelling suclavian central venous catheters. Arch Surg 1971; 102: 335. 